\title{
Progressive Cervical Myelopathy Caused by Bilateral Spontaneous High-Flow Vertebro-Vertebral Arteriovenous Fistulas Associated with Fibromuscular Dysplasia Successfully Treated by Endovascular V2 Segments Sacrifice Using Fibered Coils: A Case Report
}

\section{Prasert lampreechakul $\left(\mathbb{D}^{1}{ }^{*}\right.$, Anusak Liengudom ${ }^{1}$, Korrapakc Wangtanaphat $^{1}$, Punjama Lertbutsayanukul ${ }^{2}$, Yodkhwan Wattanasen ${ }^{2}$ and Somkiet Siriwimonmas ${ }^{3}$}

${ }^{1}$ Department of Neurosurgery, Prasat Neurological Institute, Bangkok, Thailand

${ }^{2}$ Department of Neuroradiology, Prasat Neurological Institute, Bangkok, Thailand

${ }^{3}$ Department of Radiology, Bumrungrad International Hospital, Bangkok, Thailand

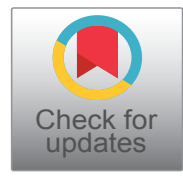

*Corresponding author: lampreechakul Prasert, Department of Neurological Surgery, Prasat Neurological Institute, 312 Rachawithi Road, Khwaeng Thung Phaya Thai, Bangkok 10400, Thailand, E-mail: bangruad@hotmail.com

\begin{abstract}
The authors reported an extremely rare case of bilateral spontaneous vertebro-vertebral arteriovenous fistulas (VVAVFs) associated with fibromuscular dysplasia (FMD). A 53-year-old hypertensive woman has been placed on antiplatelet drug following diagnosis of supratentorial ischemic stroke for 2 years. Few months before hospitalization, she developed bilateral audible bruits and progressive spastic quadriparesis. Magnetic resonance imaging (MRI) of the cervical spine showed bilateral dilated epidural venous pouches, causing cervical spinal cord compression. Angiography revealed bilateral high-flow VVAVFs with diffuse ectasia, and typical "string of beads" appearance. The left-side fistula located at C2-3 intervertebral foramen and the rightside at C3-4 intervertebral foramen. Reconstitution of bilateral vertebral arteries from bilateral occipital and ascending cervical arteries was also noted distal to the fistulous site. Other multivessel involvement included bilateral extracranial internal carotid, right renal, and intracranial arteries. The right internal carotid studies showed short segment of fenestration or duplication of the distal cervical internal carotid artery (ICA) combined with a small pseudoaneurysm at the distal end of the fenestrated segment, probably representing previous arterial dissection. Under general anesthesia, the fistulas were successfully treated by bilateral V2 segments sacrifice involving fistulous sites using fibered coils. Follow-up MRI and angiography confirmed complete obliteration of the fistulas and resolution of large epidural venous
\end{abstract}

pouches. The patient has gradually improved and returned to her normal daily activities within six months after treatment.

\section{Keywords}

Bilateral, spontaneous, vertebral arteriovenous fistula, fibromuscular dysplasia, stroke, progressive cervical myelopathy, fibered coil

\section{Introduction}

Remaining a disease of unknown etiology, pathogenesis and long- term outcome data, fibromuscular dysplasia (FMD) is non-inflammatory, non-atherosclerotic arteriopathy affecting commonly in renal, extracranial carotid, and vertebral arteries. However, FMD may affect any artery, including visceral and extremity arteries [1-7]. Multisite involvement is common [8]. The disease may manifest with a serious vascular event following nonspecific symptoms, resulting in delay in diagnosis. FMD can be identified by its characteristic angiographic appearance. In addition, histopathologic confirmation of the diagnosis of FMD was available in only small number of patients. Therefore, the diagnosis of FMD was based on angiographic or noninvasive im-

Citation: lampreechakul P, Liengudom A, Wangtanaphat $\mathrm{K}$, Lertbutsayanukul $\mathrm{P}$, Wattanasen $\mathrm{Y}$, et al. (2018) Progressive Cervical Myelopathy Caused by Bilateral Spontaneous High-Flow Vertebro-Vertebral Arteriovenous Fistulas Associated with Fibromuscular Dysplasia Successfully Treated by Endovascular V2 Segments Sacrifice Using Fibered Coils: A Case Report. Clin Med Rev Case Rep 5:248. doi. org/10.23937/2378-3656/1410248

Accepted: December 17, 2018: Published: December 19, 2018

Copyright: (c) 2018 lampreechakul P, et al. This is an open-access article distributed under the terms of the Creative Commons Attribution License, which permits unrestricted use, distribution, and reproduction in any medium, provided the original author and source are credited. 
aging $[9,10]$. According to the three main arterial wall layers, FMD is then classified into intimal, medial, and adventitial fibrodysplasia. The most common type of FMD is medial fibroplasia, representing with the typical "string of beads" appearance $[1,11]$. Vertebral artery (VA) FMD is less common than internal carotid artery (ICA) FMD and usually associated with carotid lesions
$[3,8,10,12-14]$. The rate of angiographic incidence of extracranial VA FMD is approximately $0.17 \%-0.27 \%[4,12]$. Another study of 36 patients with cervico-cephalic FMD by Pasquini, et al. [8], they found that all patients had ICA involvement, bilateral in $61 \%$ and $50 \%$ had associated VA involvement, bilateral in $44 \%$. According to the study of 70 patients with FMD by Manelfe, et al. [12],
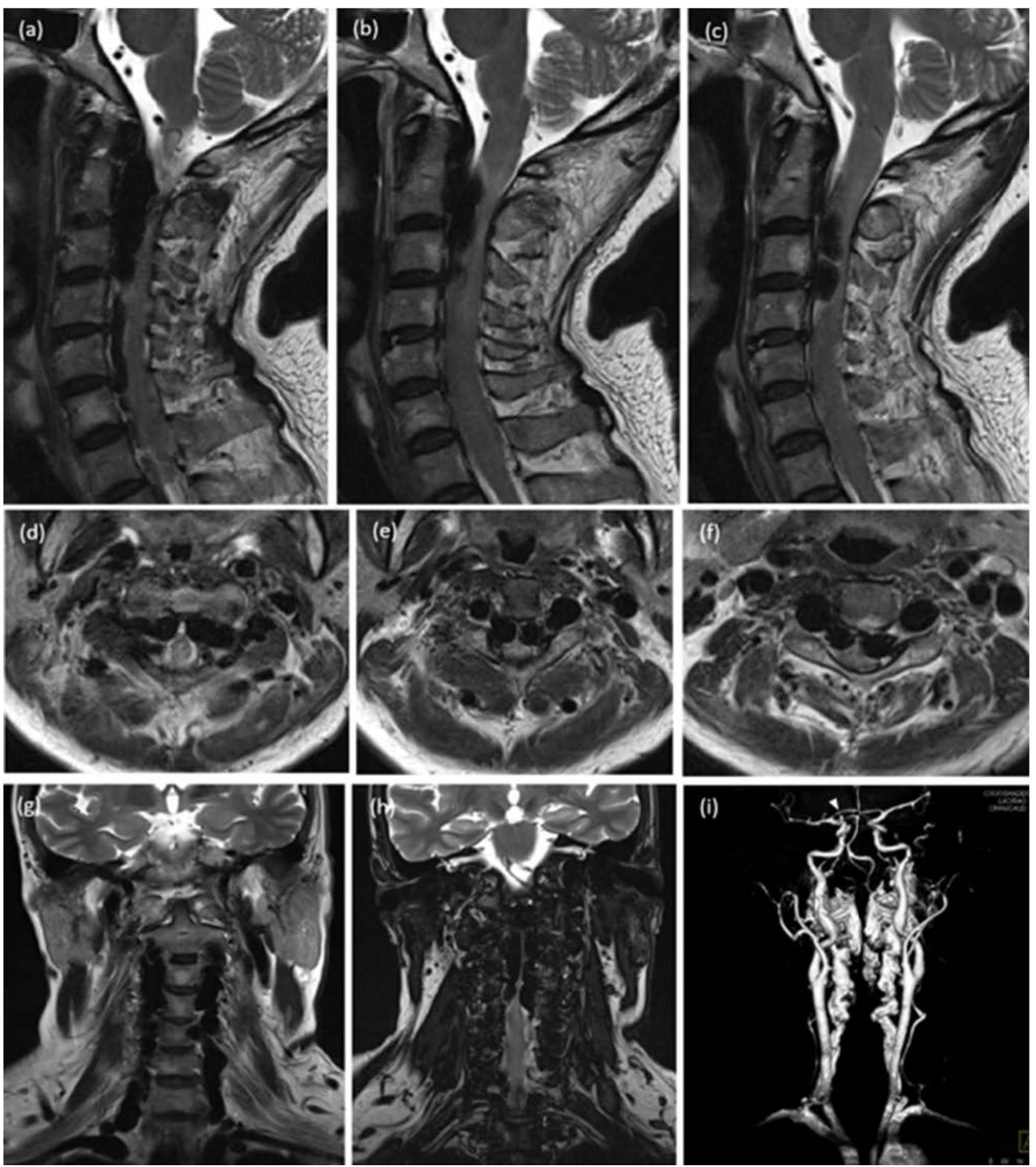

Figure 1: T2 weighted images of sequential images from right to left side of sagittal $(a, b, c)$, upper to lower level of axial (d, $e, f)$, and anterior to posterior aspect of coronal $(g, h)$ views of the cervical spine show irregularity, ectasia, and tortuosity in V1-V2 segments of both vertebral arteries, and demonstrate markedly dilatation of bilateral anterolateral epidural venous plexus as tubular flow voids of the spinal canal from C2 to C5 level, causing severe cervical cord compression. There is T2 signal hyperintensity within upper cervical cord, representing compressive myelopathy. Contrast-enhanced magnetic resonance angiographic image of the neck (i) confirms bilateral vertebro-vertebral arteriovenous fistulas and fusiform dilatation with focal dysplastic change of bilateral distal cervical internal carotid arteries (ICAs). A small saccular aneurysm of the right supraclinoid ICA is also noted (white arrowhead). 

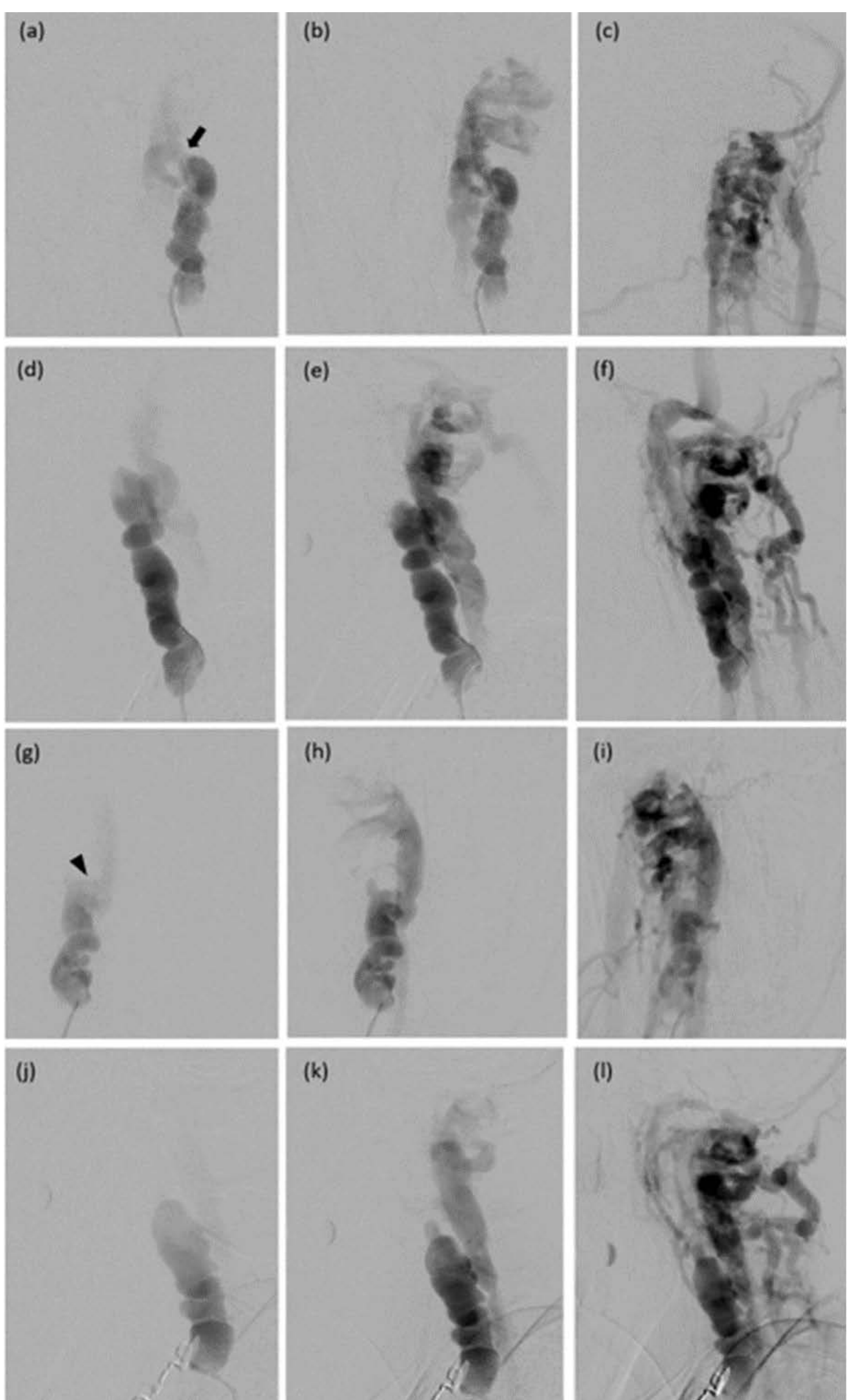

\section{(k)}

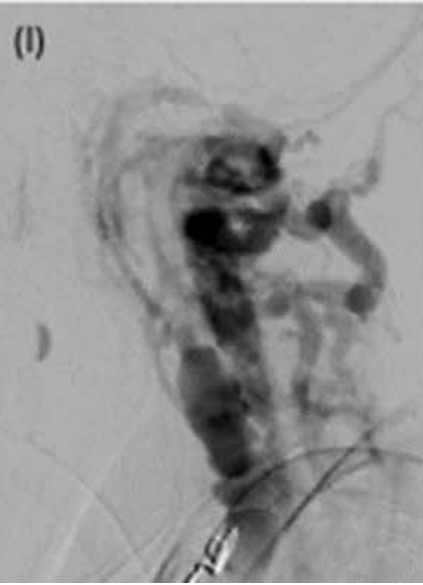

Figure 2: Anteroposterior $(a, b, c)$ and lateral $(d, e, f)$ views of the left vertebral artery (VA) injection show high-flow vertebro-vertebral arteriovenous fistula (VVAVF). There is diffuse ectasia with alternating dilatation and stenosis of V2 segment with drainage into enlarged left epidural venous plexus, posterior cervical veins, sigmoid sinus, and internal jugular vein. The fistulous site is located at C2-3 intervertebral foramen (arrow). Anteroposterior $(\mathrm{g}, \mathrm{h}, \mathrm{i})$ and lateral $(\mathrm{j}, \mathrm{k}, \mathrm{l})$ views of the right VA injection reveal high-flow VVAVF. There is diffuse ectasia with alternating dilatation and stenosis of V2 segment with drainage into enlarged right epidural venous plexus, and posterior cervical veins. The fistulous site is located at C3-4 intervertebral foramen (arrowhead). No antegrade flow of bilateral VAs is noted. 
they found VA FMD in $24 \%$ and among these, bilateral vertebral lesions at the rate of $30 \%$. The formation of arteriovenous fistula in patients with FMD may result from the rupture of the aneurysm located on dysplastic vessels, representing a complication of pre-existing arterial fibrodysplasia $[2,5,15]$.

Spontaneous vertebro-vertebral arteriovenous fistulas (VVAVFs) are non-traumatic abnormal connections between the extracranial VA or its branches and neighboring veins including epidural venous plexus, paravertebral veins, internal jugular vein, and/or perimedullary veins $[16,17]$. Spontaneous VVAVFs have been associated with FMD, Neurofibromatosis Type 1 (NF1), and Ehlers-Danlos syndrome (EDS) [18-22]. Bilateral VVAVFs are extremely rare and usually traumatic cause, especially by bilateral iatrogenic puncture $[23,24]$. Spontaneous bilateral VVAVFs associated with FMD are also extremely rare. To the best of our knowledge, only one case of bilateral VVAVFs associated with FMD has been shown previously in the data table of the series of VVAVFs by Beaujeux, et al. [17].

The authors described a patient with spontaneous bilateral VVAVFs associated with FMD manifested with cervical bruit and progressive myelopathy. The fistulas were successfully treated by bilateral V2 segments sacrifice using fibered coils.

\section{Case Report}

A 53-year-old, right-handed, non-smoker woman with medical history of hypertension and dyslipidemia was admitted to the local hospital because of right hemiparesis. She had no family history of stroke or other cerebrovascular diseases. By computed tomography (CT) scan, she was diagnosed as cerebral infarctions at left lentiform nucleus, head of caudate nucleus, internal capsule, and centrum semiovale. She had been placed on antiplatelet drug and returned to normal daily activities. Two years later, she suffered from right hemiparesis and bilateral audible bruits without history of trauma. She went to the same local hospital and was investigated by CT scan. She was diagnosed as recurrent stroke and obtained echocardiography and doppler ultrasound of both carotid arteries with results of normal study. She was discharged home 2 days later and made appointment in next one month. Two weeks later, she developed quadriparesis (power grade 3-4/5), predominantly affecting the right more than the left side, with incontinence of bowel and bladder. She went back to the hospital and was sent to our institute one month later. On physical examination, a palpable thrill and loud bruit were evident over both sides of neck. No abnormal skin lesions were detected. The neurological examination revealed evidence of spastic quadriparesis (power grade 1-2/5), the lack of pinprick sensation below C4 level, absent of proprioception, loss of anal sphincter control, hyperreflexia of upper and lower extremities, clonus, and presence of Babinski's sign.

Magnetic resonance imaging (MRI) and contrast-en-

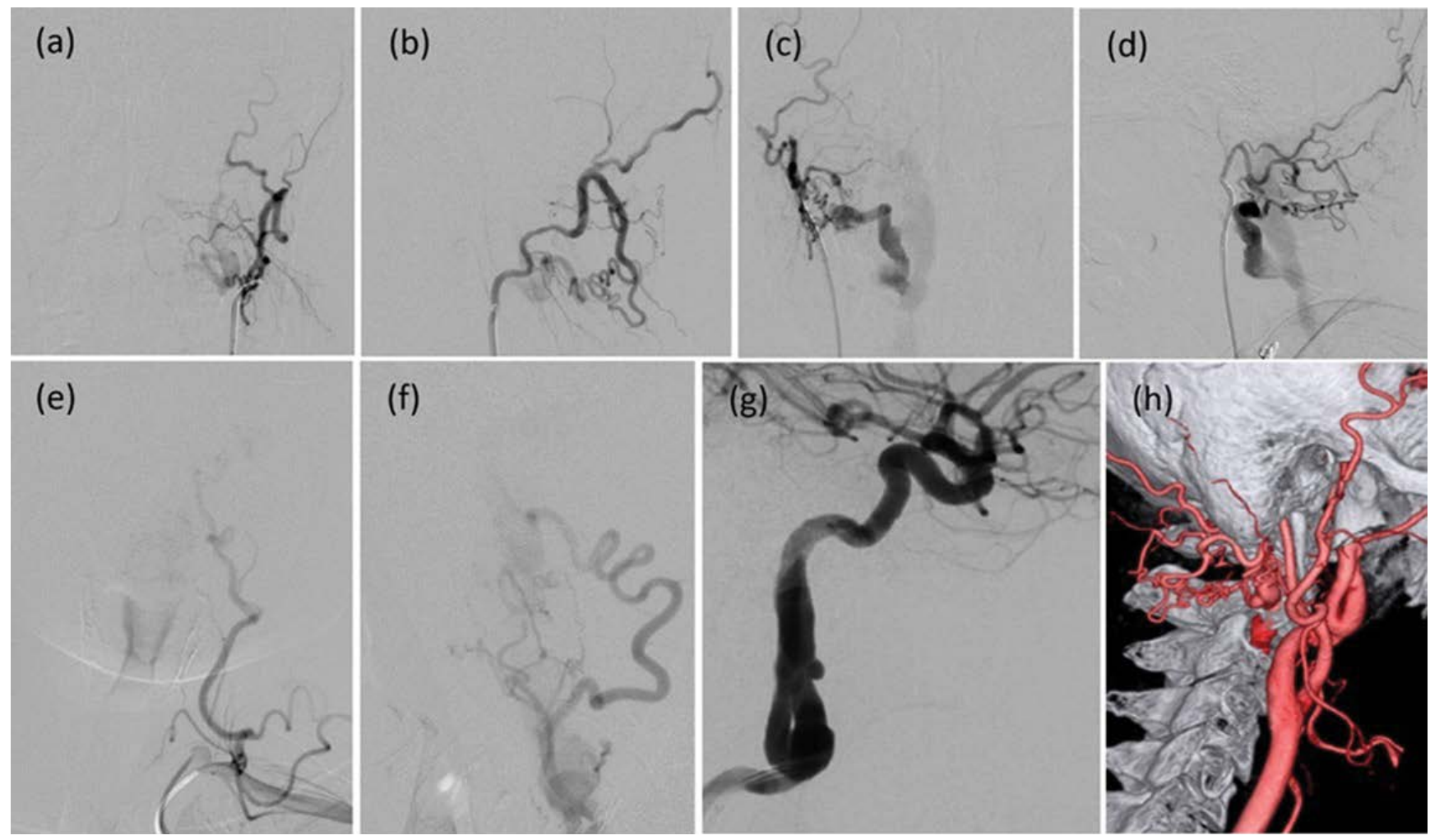

Figure 3: Anteroposterior ( $a, c)$ and lateral $(b, d)$ views of the left and right occipital arteries injections, respectively, show reconstitution of bilateral vertebral arteries (VAs). Anteroposterior (e) and lateral (f) views of the left ascending cervical artery injection also reveal reconstitution of the left VA. Lateral views $(\mathrm{g})$ and $3 \mathrm{D}$ reconstruction $(\mathrm{h})$ of the right internal carotid artery (ICA) injection demonstrate short segment fenestration or duplication of the cervical ICA combined with a small pseudoaneurysm at the distal end of the fenestrated segment. 
hanced magnetic resonance angiography (MRA) of the cervical spine showed bilateral high-flow VVAVFs. Enormous dilatation, markedly tortuosity, and irregularity of the V1-V2 segments of both vertebral arteries were apparent. Abnormal enlarged epidural vertebral venous plexus as tubular flow voids of the spinal canal from C1-C5 level on both sides compressed severely anterolateral aspect of cervical cord, especially at C2-C3 level. There was swelling of spinal cord from C1-C7 with abnormal hypersignal T2 change of the spinal cord from C2-C4, representing myelopathy (Figure 1). In addition, MRA of renal arteries showed dysplastic change and stenosis of the right side.
Digital subtraction angiography (DSA) revealed bilateral high-flow VVAVFs with diffuse ectasia, and alternating areas of dilatation and stenosis of bilateral V1-V2 segments, representing typical "string of beads" appearance. The left-side fistula located at C2-3 intervertebral foramen and drained directly into enlarged left anterior internal (epidural) vertebral venous plexus with another route draining into left posterior cervical veins and internal jugular vein, subsequently upward draining into left sigmoid sinus and suboccipital veins. The right-side fistula located at C3-4 intervertebral foramen and drained directly into dilated right anterior internal (epidural) vertebral venous plexus with another route draining into right internal jugular and posteri-
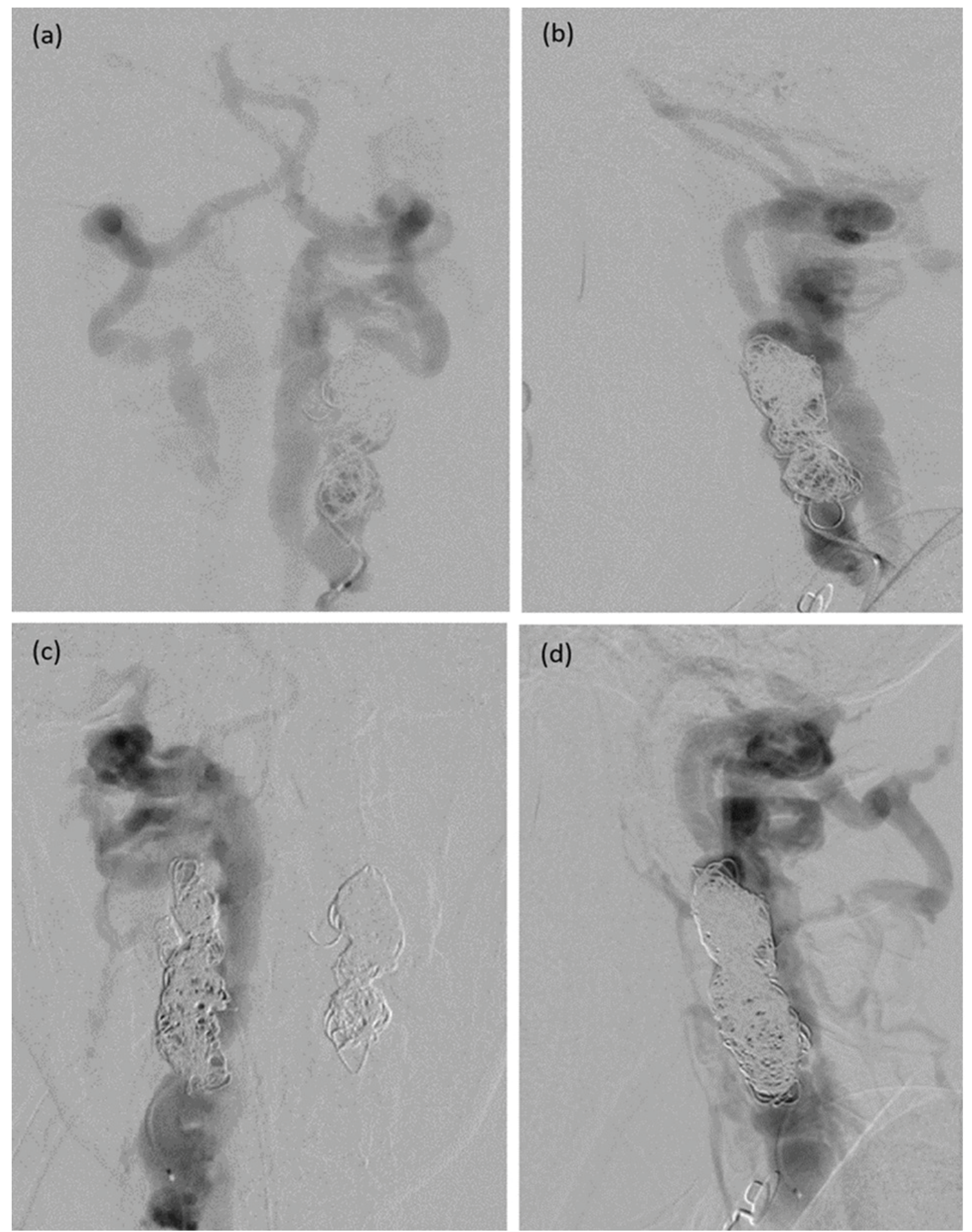

Figure 4: Anteroposterior (a, c) and lateral (b, d) views of the left and right vertebral arteries (VAs) injections, respectively, after packing with fibered coils at fistulous site and V2 segments proximal to the fistulas show residual shunts with antegrade flow of both VAs. 
or cervical veins. No antegrade filling of distal VAs was seen (Figure 2). Reconstitution of bilateral VAs from bilateral occipital and ascending cervical arteries was also noted distal to the fistulous site (Figure 3a, Figure 3b, Figure 3c, Figure 3d, Figure $3 e$ and Figure 3f). The right internal carotid studies showed short segment of fenestration or duplication of the distal cervical ICA combined with a small pseudoaneurysm at the distal end of the fenestrated segment (Figure $3 \mathrm{~g}$ and Figure $3 \mathrm{~h}$ ). A small saccular aneurysm was detected at superior aspect of the right clinoid ICA. Fusiform dilatation and mild focal dysplastic change of bilateral distal cervical ICAs were observed.

Under general anesthesia with bilateral femoral approaches, the left V2 segment occlusion was performed at the fistulous site and proximal portion to the fistula with 10 detachable fibered coils (Interlock ${ }^{\mathrm{TM}}-35$, Boston Scientific, Natick, MA). The same procedure was then performed at the right $\mathrm{V} 2$ segment with 22 fibered coils. The size of fibered coils used for embolization in our case varied from $5 \mathrm{~mm} \times 15 \mathrm{~cm}$ to $12 \mathrm{~mm} \times 30 \mathrm{~cm}$. Immediate postembolization, DSA revealed residual shunt at both sides but significant reduction of flow with antegrade filling of distal VAs was seen (Figure 4).

One day after treatment, the audible bruits and neck thrill disappeared, and power grade of the patient improved to grade $2-3 / 5$. The patient gradually improved following continuous rehabilitation program. MRI follow-up one-month after embolization showed disappearance of bilateral dilated epidural venous plexus
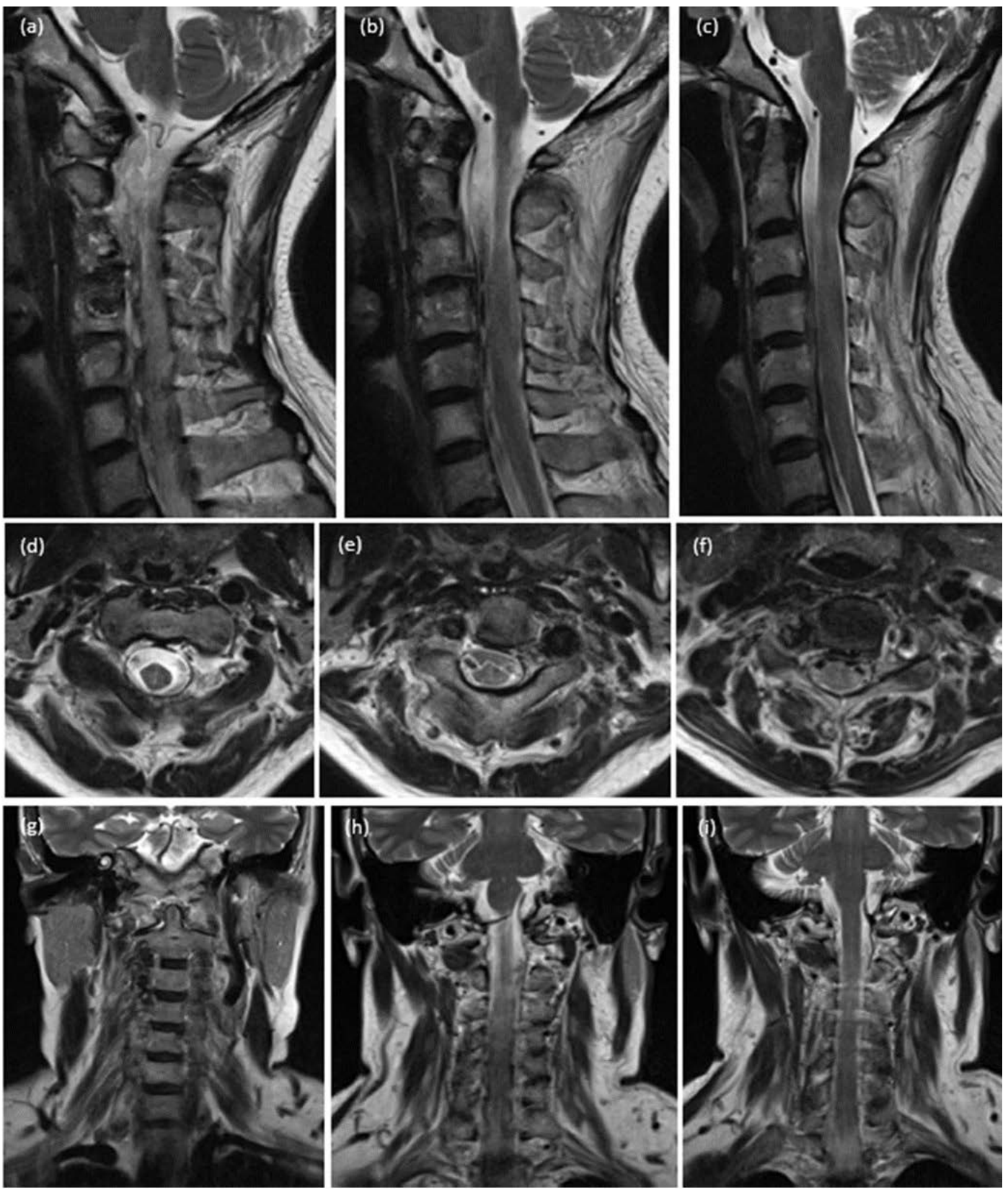

Figure 5: One month after embolization, T2 weighted images of sequential images from right to left side of sagittal (a, b, c), upper to lower level of axial (d, e, f), and anterior to posterior aspect of coronal $(\mathrm{g}, \mathrm{h}, \mathrm{i})$ views of the cervical spine reveal disappearance of bilateral dilated epidural venous plexus with resolution of cervical cord compression. Enlarged bilateral vertebral arteries are still observed with T2 hyperintensity instead of T2 hypointensity from previous study, suggesting flow reduction. 
with resolution of cervical cord compression. Enlarged bilateral VAs were still observed with hyperintense on T2-weighted MRI sequence instead of hypointense from previous study, suggesting flow reduction of the fistulas (Figure 5). Follow-up DSA, obtained three months after embolization, revealed residual shunt on the left side and complete obliteration of the right fistula. There was significant regression of the irregular enlarged VAs. Reconstitution of the VAs distal to the site of embolization through occipital and enlarged ascending cervical arteries was noted (Figure 6). The patient got back to her normal daily activities with full recovery of bowel and bladder functions six months after treatment. Two years after embolization, follow-up DSA confirmed no recurrent of the fistulas with residual right VA proximal to the pack of coils. There was a good collateral circulation from bilateral occipital and ascending cervical arteries (Figure 7).

\section{Discussion}

Regarding venous drainage of the VVAVF, the fistula directly drained into the anterior and/or posterior internal vertebral venous plexus (epidural). Intervertebral vein, communicating with internal vertebral venous plexus, may be distended by shunting and exited through cervical neural foramen to paravertebral veins and external venous plexus, including the suboccipital venous system, internal jugular vein, and/or deep cervical veins. Retrograde venous drainage into medullary system (intradural) drained via the bridging vein between intervertebral vein and radicular vein, running along the cervical nerve root [25].

The mechanism of myelopathy in VVAVFs can result from direct spinal cord compression from epidural venous engorgement, and/or venous hypertension from pial venous reflux $[18,26]$. Undoubtfully, the mechanism of severe cervical cord compression in our
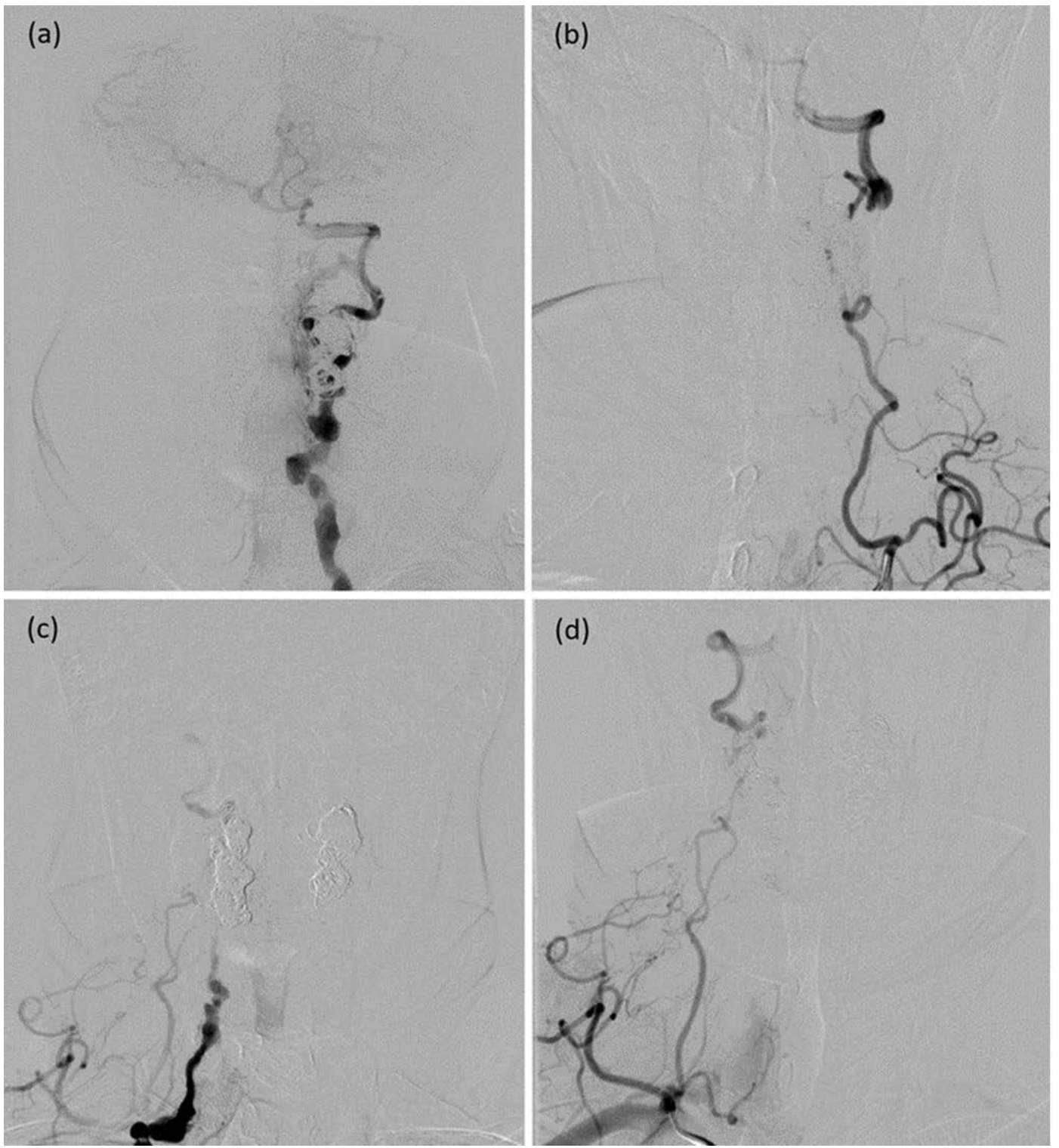

Figure 6: Three months after embolization, anteroposterior views of the left (a) and right (C) vertebral arteries (VAs) and left (b) and right (d) thyrocervical trunks injections reveal residual shunt on the left side and complete obliteration of the right fistula. There has been significantly regression of the irregular enlarged VAs. Reconstitution of the VAs distal to the site of embolization through enlarged ascending cervical arteries is noted. 

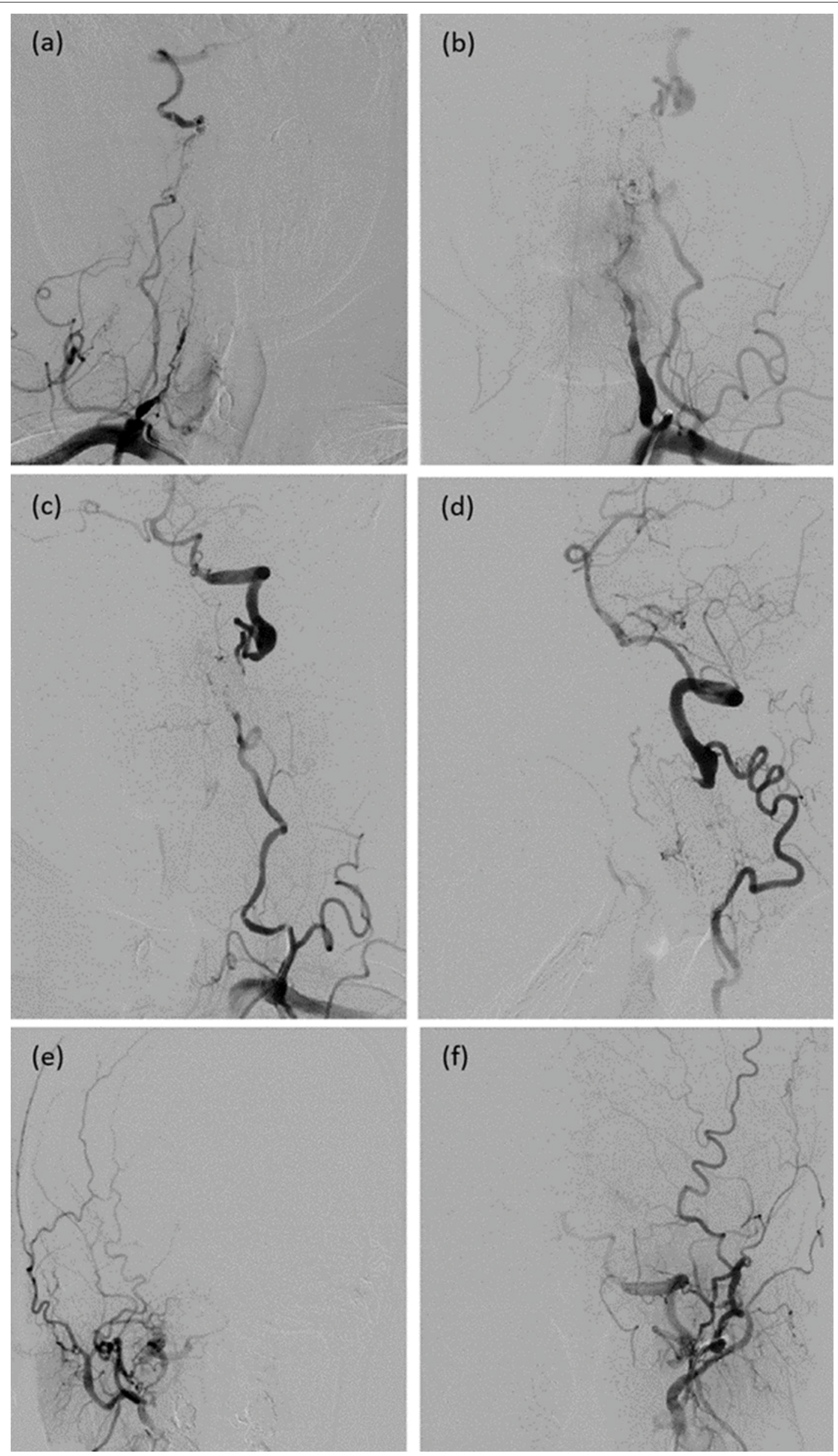

Figure 7: Two years after embolization, anteroposterior $(a, c)$ and lateral $(b, d)$ views of the right and left subclavian arteries injections, respectively, confirm no recurrent of the fistulas with residual right vertebral artery (VA) proximal to the pack of coils. There is good collateral circulation from hypertrophic ascending cervical arteries. Anteroposterior views of the right (e) and left (f) occipital arteries also reveal reconstitution of bilateral VAs. 
case can occur from forceful epidural compression from bilateral dilated epidural venous pouches. The large epidural venous pouch compressing spinal cord from spontaneous VVAVF was found more frequently in the fistula associated with NF1 [21,26-31].

Bilateral VVAVFs are extremely rare. They can be caused by trauma or may occur spontaneously. For traumatic cause, bilateral VVAVFs may result from iatrogenic puncture of both VAs [23,24]. Most of these fistulas tend to resolve spontaneously because of the small size of the fistulas. Bilateral spontaneous VVAVFs have rarely been reported in EDS, NF1, and FMD $[17,20,26,27]$. Goyal, et al. [20] reported bilateral VVAVFs at the level of $\mathrm{C} 2$ and $\mathrm{C} 4$ in the patient associated with EDS. Due to asymptomatic fistulas, they left untreated. The first case of bilateral VVAVFs associated with NF1 was reported by Hasegawa, et al. [26] They speculated that the atlantoaxial dislocation probably plays an important role in the formation of the bilateral VVAVFs. Only left-sized fistula was successfully treated by a combination of balloon embolization and surgery followed by occipitocervical fusion. Subsequently, Siddhartha, et al. [27] reported another similar case of bilateral VVAVFs associated with NF1 presenting with neck pain and quadriparesis. At the level of $C 5$ bilaterally, the left fistula and VA were successfully occluded with balloons following successful occlusion of the right fistula and VA with coils in separate sessions at 2 days interval without test occlusion due to reformation from bilateral ascending cervical, ascending pharyngeal, and occipital arteries. The patient improved significantly and able to walk with support at 6 months follow-up. In series of 35 VVAVFs in 34 patients of various causes reported by Beaujeux, et al. [17], they found one patient of bilateral spontaneous VVAVFs associated with FMD presenting with tinnitus. Both fistulas were located at the level of $C 1$ to $C 2$ and successfully treated with balloons embolization. Other data in this patient were not available in the published study.

Rarely, the patients with VVAVF may develop an embolic posterior circulation stroke, producing vertigo, ataxia, or homonymous hemianopia [32,33]. Thromboembolism may result from abnormal flow pattern within the venous outlet, producing reflux of venous clot, leading to stroke in posterior fossa. Similarly, John, et al. [32] reported a case of spontaneous VVAVF causing acute ischemic stroke and cervical myelopathy. However, the stroke with right hemiparesis in our case was compatible with an anterior circulation or supratentorial stroke, probably related to dysplastic changes of the left ICA.

Based on 6 angiographic observations of apparent fenestration of the cervical ICA, Gailloud, et al. [34] have termed "pseudofenestration", i.e. short segment fenestration or duplication of the cervical ICA, and strongly suggested that this condition probably was a sequela of a segmental arterial dissection with a double lumen sign rather than a congenital anatomical variant. Interestingly, they also found that 3 out of 6 patients in their series had angiographic features of FMD. Thromboembolic events may relate to pseudofenestration of the cervical ICA [35]. Therefore, we believe that short segment fenestration of the right cervical ICA combined with a pseudoaneurysm at the distal end of the fenestrated segment in our case were the pseudofenestration secondary to previous arterial dissection on segment of ICA FMD.

According to the United States (US) registry for FMD in 447 patients by Olin, et al. [10], FMD occurred primarily in middle-aged woman and commonly affects the renal, extracranial carotid, and VAs. Of 357 patients obtained at least 2 vascular beds imaged, they found that $35.3 \%$ had FMD in 2 vascular beds, representing common multivessel involvement. Hypertension, headache, pulsatile tinnitus, and dizziness are the most common presenting symptoms of this disease. There was a significant delay in diagnosis from the first onset of clinical symptoms and signs of 4.1 years in woman. These patients also had a transient ischemic attack or stroke with a high frequency. The most common clinical presentation of renal artery FMD is hypertension in young woman [14]. Therefore, stroke in young and middle-aged with hypertension should be investigated for FMD by non-invasive imaging in cervico-cephalic and renal arteries. In addition, the patients with cervico-encephalic FMD should be obtained computed tomography angiography (CTA) or MRA of renal arteries for diagnostic confirmation [6].

Subsequently, after 921 patients enrolled in the US FMD registry, the study revealed that these patients have high prevalence $(41.7 \%$ ) of aneurysm (common in the extracranial carotid, renal, and intracranial arteries) and/or dissection (common in the extracranial cervical, renal, and coronary arteries) prior to or at the time of FMD diagnosis [7]. However, this largest series of FMD did not mention about arteriovenous fistula of involving vessels, confirming being extremely rare condition. In addition, the diagnosis and treatment of VVAVF associated with FMD may be delayed, leading to potentially serious sequelae as in the current case which was delayed in diagnosis of FMD for 2 years following first symptoms of stroke.

Pasquini, et al. [8] studied the prevalence of multisite involvement and prognosis in 36 patients with cervico-cephalic FMD and found that renal artery FMD was present in $43 \%$ of patients. They disagreed that FMD is usually considered as a relative benign disease because the risk of recurrent stroke, death, or FMD progression was high. From the meta-analysis in 498 patients with FMD involving ICA and/or VA by Cloft, et al. [36], the prevalence of incidental, asymptomatic intracranial saccular aneurysms was approximately $7 \%$. We also found an unruptured intracranial aneurysm in the current case. 
Varennes, et al. [6] have demonstrated the less common imaging findings of FMD, including vascular loops, fusiform vascular ectasia, arterial dissection, aneurysm, and subarachnoid hemorrhage. In the present case, we also found fusiform vascular ectasia of both carotid arteries, pseudofenestration of the right cervical ICA, and intracranial aneurysm of the right ICA. FMD usually affects middle and distal portions of internal carotid and vertebral arteries $[5,9]$. Interestingly, in the present case, VA FMD also involved bilateral proximal portions.

The goal of the treatment of the VVAVF is the obliteration of the fistula and preservation of VA flow. However, preservation of the VA is often not possible [20]. Proximal occlusion or trapping procedure without obliteration of the fistula may be ineffective because collateral vessels, i.e. segmental branches of VA, ascending cervical, deep cervical, ascending pharyngeal, and/or occipital arteries, may maintain the fistula patency $[16,22,24]$.

Initially, the obliteration of the VVAVFs by endovascular treatment with preservation of vertebral flow or trapping procedure can be performed mainly by detachable balloons $[16,17]$. Occasionally, the fistula requires a combination of endovascular treatment and surgery for achieving completely obliteration. By surgical treatment alone, it is difficult, and often unsatisfactory because of uncontrollable massive bleeding from the engorged veins. Due to unpredictable complication, such as early deflation of the balloon, coiling and stenting have been increasingly used as an alternative treatment in recent decades. In addition, it is difficult to navigate balloon catheter through tortuous and irregular arteries involved by FMD $[15,17,26,28]$. According to anatomically based approach for embolization of VVAVFs by Yeh, et al. [37], they proposed that the fistula in V1-V2 segments of the VA could be treated effectively by covered stent with preserving of the patency of the VA. Stent graft may be used in a single large hole and high-flow VVAVF with no apparent difference between the proportions of the proximal VA and distal to the fistula site [28]. For the fistula associated with FMD, however, it is also difficult to navigate the stent through tortuosity of the vessels.

Generally, endovascular treatment of large VVAVFs

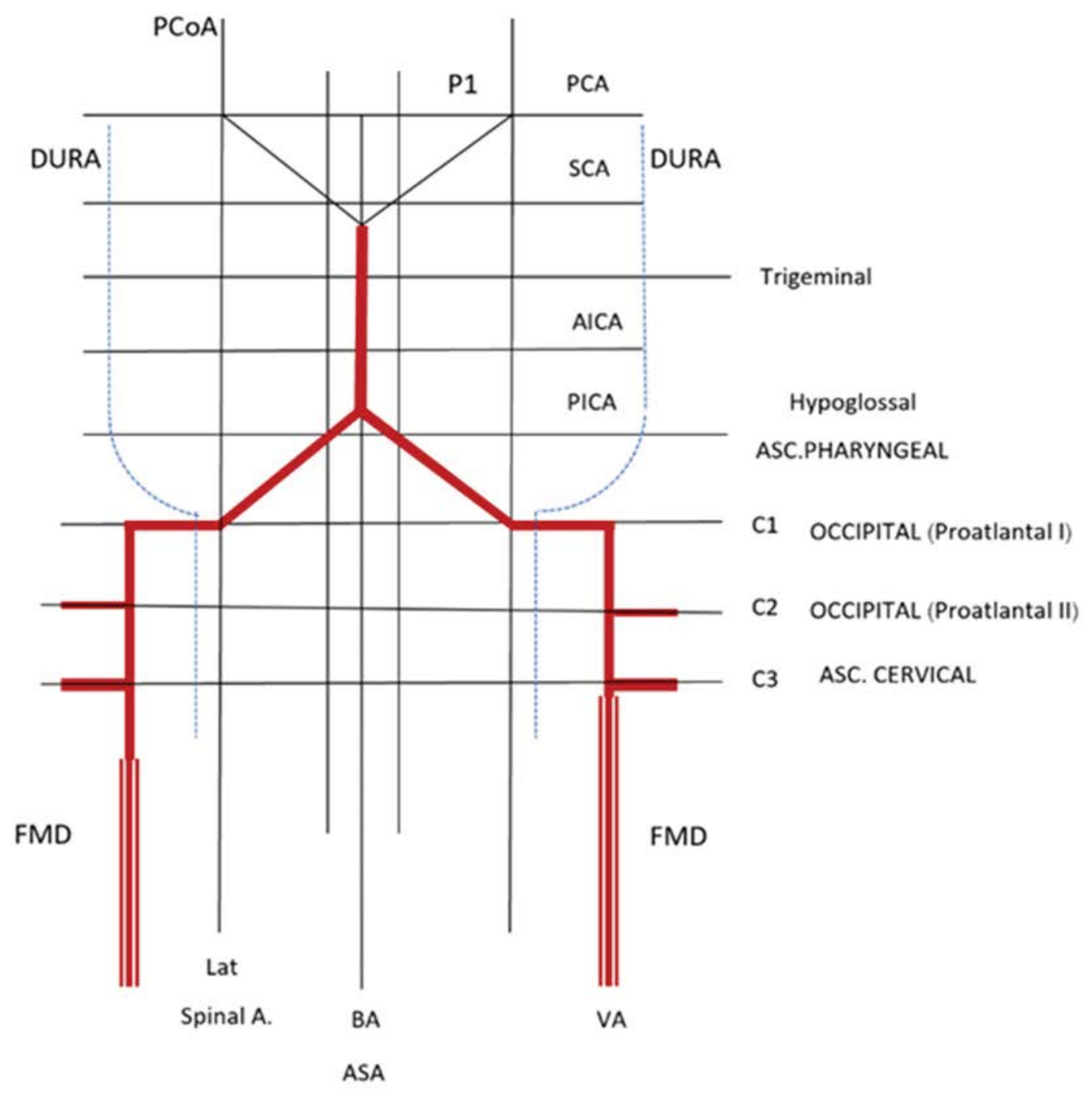

Figure 8: Schematic illustration of the vertebrobasilar system superimposed on a grid representing fibromuscular dysplasia (FMD) of bilateral vertebral arteries (VAs) with arterial variations, including bilateral occipital-vertebral anastomoses at level of $\mathrm{C} 2$ and bilateral ascending cervical-vertebral anastomoses at level of C3. (Adapted from Lasjaunias P, et al. [38]). 
with liquid embolic materials should be avoided due to high risk of distal embolic events from high-flow fistula. N-butyl cyanoacrylate (NBCA) may be injected to completely occlude the fistula following packing of coils [22]. Recently, the VVAVF with drainage into large epidural venous pouch may be treated by endovascular balloon-assisted coiling and Ethylene vinyl alcohol (EVOH) embolization [32]. However, myelopathic symptoms may be delayed in improvement by this technique because of remaining mass effect from coils and liquid embolic materials inside large venous lake. By technique of closure of the fistulas and VAs sacrifice as in our case, MRI of cervical spine one month after embolization revealed complete resolution of the epidural venous enlargement and rapid resolution of myelopathic symptoms occurred within few months.

VA sacrifice in spontaneous VVAVFs is often necessary but well tolerated [20]. Similar to extracranial fistula in NF1 patients, the optimal treatment for VVAVFs in FMD is sacrifice of the affected artery due to the pathologic changes in the arterial wall. Complete assessment of the angioarchitecture or vascular anatomy of the VVAVFs and identification of the collateral flow from the uninvolved VA is imperative before planning endovascular treatment [21].

Adapted from Lasjaunias, et al. [38], arterial variations in our case were plotted on a diagrammatic representation of the vertebrobasilar system (Figure 8). In occlusive conditions of the VAs, a potential source of collateral circulation may be occurred from the anastomoses at the level of $\mathrm{C} 1$ to $\mathrm{C} 4$. Common extradural variations of the VA include occipito-vertebral anastomoses at the level of C1 to C2 (embryonic arterial remnants of proatlantal I and II), and the ascending cervical artery entering the vertebral canal at the level of C3 to C4. In addition, another anastomotic filling of the distal ipsilateral VA may arise from the ascending pharyngeal artery $[21,27,31]$.

Without surgical decompression, few case reports including our case demonstrated that the huge dilated venous structures resolved following the occlusion of fistulous site and parent artery with coils and/or balloon [25,31]. Furthermore, bilateral obliteration of the fistulas and VAs sacrifice in our case can achieve in the same session.

In order to promote thrombus formation, we preferred to use fibered coils in the present case because fibered coils are more thrombogenic than bare coils, leading to significantly improved obliteration rates [39]. Serial follow-up vertebral angiography in our case revealed gradually resolution of the fistulas until complete closure, confirming further thrombogenicity of fibered coils.

In the present case, FMD manifesting with a serious vascular event following 2 years of continuous antiplate- let therapy after mild stroke symptoms supported poor prognosis in the FMD patients. However, the disabled patient can return to a normal life with good outcome following prompt endovascular treatment.

\section{Conclusion}

The authors illustrated an extremely rare case of bilateral spontaneous VVAVFs associated with FMD involving multivessel. Compressive cervical myelopathy, leading to progressive deterioration of symptoms, caused by forceful bilateral enlarged epidural venous lakes. By endovascular treatment with fibered coils, we have achieved complete closure of the fistulas and parent VAs with excellent outcome.

\section{References}

1. Begelman SM, Olin JW (2000) Fibromuscular dysplasia. Curr Opin Rheumatol 12: 41-47.

2. Bellot J, Gherardi R, Poirier J, Lacour P, Debrun G, et al. (1985) Fibromuscular dysplasia of cervico-cephalic arteries with multiple dissections and a carotid-cavernous fistula. A pathological study. Stroke 16: 255-261.

3. Mettinger KL (1982) Fibromuscular dysplasia and the brain. II. Current concept of the disease. Stroke 13: 53-58.

4. Stanley JC, Fry WJ, Seeger JF, Hoffman GL, Gabrielsen TO (1974) Extracranial internal carotid and vertebral artery fibrodysplasia. Arch Surg 109: 215-222.

5. Touzé E, Oppenheim C, Trystram D, Nokam G, Pasquini $M$, et al. (2010) Fibromuscular dysplasia of cervical and intracranial arteries. Int J Stroke 5: 296-305.

6. Varennes L, Tahon F, Kastler A, Grand S, Thony F, et al. (2015) Fibromuscular dysplasia: What the radiologist should know: A pictorial review. Insights Imaging 6: 295307.

7. Kadian-Dodov D, Gornik HL, Gu X, Froehlich J, Bacharach JM, et al. (2016) Dissection and aneurysm in patients with fibromuscular dysplasia: Findings from the U.S. registry for FMD. J Am Coll Cardiol 68: 176-185.

8. Pasquini M, Trystram D, Nokam G, Gobin-Metteil MP, Oppenheim C, et al. (2015) Fibromuscular dysplasia of cervicocephalic arteries: Prevalence of multisite involvement and prognosis. Rev Neurol (Paris) 171: 616623.

9. Osborn AG, Anderson RE (1977) Angiographic spectrum of cervical and intracranial fibromuscular dysplasia. Stroke 8: 617-626.

10. Olin JW, Froehlich J, Gu X, Bacharach JM, Eagle K, et al. (2012) The United States registry for fibromuscular dysplasia: Results in the first 447 patients. Circulation 125 : 3182-3190.

11. Stanley JC, Gewertz BL, Bove EL, Sottiurai V, Fry WJ (1975) Arterial fibrodysplasia. Histopathologic character and current etiologic concepts. Arch Surg 110: 561-566.

12. Manelfe C, Clarisse J, Fredy D (1974) Fibromuscular dysplasia of the cervico-cephalic arteries: Report of 70 cases. J Neuroradiol 1: 149-231.

13. Houser OW, Baker HL Jr, Sandok BA, Holley KE (1971) Cephalic arterial fibromuscular dysplasia. Radiology 101: 605-611.

14. Olin JW, Sealove BA (2011) Diagnosis, management, and 
future developments of fibromuscular dysplasia. $\mathrm{J}$ Vasc Surg 53: 826-836.

15. Bahar S, Chiras J, Carpena JP, Meder JF, Bories J (1984) Spontaneous vertebro-vertebral arterio-venous fistula associated with fibro-muscular dysplasia. Report of two cases. Neuroradiology 26: 45-49.

16. Halbach VV, Higashida RT, Hieshima GB (1988) Treatment of vertebral arteriovenous fistulas. AJR Am J Roentgenol 150: 405-412.

17. Beaujeux RL, Reizine DC, Casasco A, Aymard A, Rüfenacht $D$, et al. (1992) Endovascular treatment of vertebral arteriovenous fistula. Radiology 183: 361-367.

18. lampreechakul P, Siriwimonmas S (2016) Spontaneous obliteration of spontaneous vertebral arteriovenous fistula associated with fibromuscular dysplasia after partial surgery: A case report. Interventional Neuroradiology 22: 717-727.

19. Reddy SV, Karnes WE, Earnest F 4th, Sundt TM Jr (1981) Spontaneous extracranial vertebral arteriovenous fistula with fibromuscular dysplasia. Case report. J Neurosurg 54 399-402.

20. Goyal M, Willinsky R, Montanera W, Terbrugge K (1999) Spontaneous vertebrovertebral arteriovenous fistulae clinical features, angioarchitecture and management of twelve patients. Interv Neuroradiol 5: 219-224.

21. Pereira VM, Geiprasert S, Krings T, Caldas JG, Toulgoat $\mathrm{F}$, et al. (2007) Extracranial vertebral artery involvement in neurofibromatosis type I. Report of four cases and literature review. Interv Neuroradiol 13: 315-328.

22. Hori Y, Goto K, Ogata N, K Uda (2000) Diagnosis and endovascular treatment of vertebral arteriovenous fistulas in neurofibromatosis type 1. Interv Neuroradiol 6: 239-250.

23. Bergquist E (1971) Bilateral arteriovenous fistulae. A complication of vertebral angiography by direct percutaneous puncture. Two cases, one with spontaneous closure. Br J Radiol 44: 519-523.

24. Newton TH, Darroch J (1966) Vertebral arteriovenous fistula complicating vertebral angiography. Acta Radiol Diagn (Stockh) 5: 428-440.

25. Tse GH, Patel UJ, Coley SC, Dyde RA (2017) Cervical cord decompression following embolisation of a giant cervical vertebro-vertebral arteriovenous fistula. Interv Neuroradiol 23: 399-404.

26. Hasegawa H, Bitoh S, Katoh A, Tamura K (1989) Bilateral vertebral arteriovenous fistulas and atlantoaxial dislocation associated with neurofibromatosis--case report. Neurol Med Chir (Tokyo) 29: 55-59.

27. Siddhartha W, Chavhan GB, Shrivastava M, Limaye US (2003) Endovascular treatment for bilateral vertebral arteriovenous fistulas in neurofibromatosis 1. Australas
Radiol 47: 457-461.

28. Baltacioğlu F (2009) Endovascular treatment of a vertebral arteriovenous fistula: Case report. Marmara Medical Journal 22: 248-251.

29. Patro SN, Gupta AK, Arvinda HR, Jolapara MB, Saini J (2009) Combined transarterial and percutaneous coiling of a spontaneous vertebrovertebral fistula associated with neurofibromatosis Type 1. Case report. J Neurosurg 111: 37-40.

30. Cluzel P, Pierot L, Leung A, Gaston A, Kieffer E, et al. (1994) Vertebral arteriovenous fistulae in neurofibromatosis: Report of two cases and review of the literature. Neuroradiology 36: $321-325$.

31. Johnson CE, Russell EJ, Huckman MS (1990) Resolution of spinal epidural vascular pseudotumor following balloon occlusion of a postoperative vertebral arteriovenous fistula. Neuroradiology 31: 529-532.

32. John S, Jaffari N, Lu M, Hussain MS, Hui F (2014) Spontaneous vertebral arteriovenous fistula causing cervical myelopathy and acute ischemic strokes treated by endovascular balloon-assisted coiling and Onyx embolization. J Clin Neurosci 21: 167-170.

33. Adams A, Allouni K, Basheer S, Evanson J, Mankad K (2013) Case report: Spontaneous resolution of an established iatrogenic vertebral arteriovenous fistula. J Child Neurol 28: 255-258.

34. Gailloud P, Carpenter J, Heck DV, Murphy KJ (2004) Pseudofenestration of the cervical internal carotid artery: A pathologic process that simulates an anatomic variant. AJNR Am J Neuroradiol 25: 421-424.

35. Nakamura $\mathrm{H}$, Yamada $\mathrm{H}$, Nagao T, Fujita K, Tamaki $\mathrm{N}$ (1993) Fenestration of the internal carotid artery associated with an ischemic attack--case report. Neurol Med Chir (Tokyo) 33: 306-308.

36. Cloft HJ, Razack N, Kallmes DF (1999) Prevalence of cerebral aneurysms in patients with persistent primitive trigeminal artery. J Neurosurg 90: 865-867.

37. Yeh CH, Chen YL, Wu YM, Huang YC, Wong HF (2014) Anatomically based approach for endovascular treatment of vertebro-vertebral arteriovenous fistula. Interv Neuroradiol 20: $766-773$.

38. Lasjaunias P, Berenstein A, Ter Brugge KG (2004) The pharyngo-occipital system. In: Lasjaunias $P$, Berenstein A, Ter Brugge KG, Surgical neuroangiography. Volume 1, Springer-Verlag, New York, 165-174.

39. Liebig T, Henkes H, Fischer S, Weber W, Miloslavski E, et al. (2004) Fibered electrolytically detachable platinum coils used for the endovascular treatment of intracranial aneurysms. Initial experiences and mid-term results in 474 aneurysms. Interv Neuroradiol 10: 5-26. 